\title{
SOLITONS IN A ONE-DIMENSIONAL DEGENERATE HUBBARD MODEL
}

\author{
S. LIPIŃSKI \\ Institute of Molecular Physics, Polish Academy of Sciences \\ Smoluchowskiego 17/19, 60-179 Poznani, Poland \\ (Received March 15, 1991; in final version July 24, 1991)

\begin{abstract}
An effective magnon-lattice Hamiltonian for the degenerate Hubbard chain with electron-phonon interaction is derived and a formalism for the description of solitary magnons is presented.
\end{abstract}

PACS numbers: $71.38 .+i, 71.10 .+x$

\section{Introduction}

Over the past few years many papers have been published on the one-dimensional systems dealing with the question whether equation of motion for them admit the existence of soliton solutions (e.g.[1-9]). One of the possible sources of nonlinearity is the electron-phonon coupling. Pushkarov et al. [4] considered a soliton formation in a ferromagnetic Heisenberg chain caused by the magnon-phonon coupling. The present paper extends the problem into itinerant system. The following discussion is stimulated by a recent discovering of quasi one-dimensional organic ferromagnets [10] which are believed to be well described by the one-dimensional doubly degenerate Hubbard model (DDH) [11]. It is well-known that molecular crystals are good candidates for the occurence of solitons. The widely discussed Davydov soliton [12] describing the transport of intramolecular energy and electrons along $\alpha$-helical protein molecules is the best known example.

Molecular crystals are mechanically soft (small elasticity coefficient) and due to the sensitivity of $\pi$-orbital overlap on the local distortion a strong electron-phonon coupling is expected.

The aim of the present paper is to give a general schema of looking for the soliton solution in itinerant ferromagnets and DDH is chosen only because it is the simplest itinerant model having a ferromagnetic Hartree-Fock ground state $[13,14]$. A complex question of whether these solutions represent stable 
nonlinear excitations of the one-dimensional degenerate Hubbard model coupled to the lattice will be examined in a subsequent paper [19].

\section{Model}

We consider the doubly degenerate Hubbard chain coupled to the harmonic lattice

$$
H=H_{\mathrm{e}}+H_{\mathrm{L}}+H_{\mathrm{e}-\mathrm{L}}
$$

where

$$
\begin{aligned}
& H_{\mathrm{e}}=\sum_{i \lambda \sigma} t\left(c_{i+1, \lambda \sigma}^{+} c_{i \lambda \sigma}+\text { h.c. }\right)+\sum_{i \lambda} U n_{i \lambda+} n_{i \lambda}+\sum_{i \sigma \sigma} V n_{i 1 \sigma} n_{i 2 \sigma^{\prime}} \\
& -\sum_{i \sigma \sigma^{\prime}} J c_{i 1 \sigma^{\prime}}^{+} c_{i 1 \sigma^{\prime}} c_{i 2 \sigma^{\prime}}^{+} c_{i 2 \sigma}
\end{aligned}
$$

where $c_{i \lambda \sigma}^{+}$is the electron creation operator at the site $i$ in the $\lambda$ orbital $(\lambda=1,2)$ with spin $\sigma$. The parameter $t$ is a hopping integral between nearest-neighbor sites, and we assume that the two $\lambda$ orbitals are not mixed by the hopping. The second term, proportional to $U$, represents the intraorbital Coulomb repulsion. The third term, proportional to $V$, describes the interorbital Coulomb repulsion and the final term containing $J$ represents the exchange interaction.

In the degenerate model it is possible to have an ordering of the orbital states besides the magnetic order [14-18]. From now on, we will consider the average electron density per site $n$ not much different from unity, what corresponds to the occupation in the earlier mentioned ferromagnetic organic systems $[10,11]$. In the strong coupling limit at $T=0$ the ground state for $n=1$ is ferromagnetic with simultaneous orbital order of "antiferromagnetic" type $[14,15]$. For $n \neq 1$ ferromagnetic ordering with no orbital order is also possible (high values of $J$ are not favourable to an orbital superlattice [19]). The lattice part of the Hamiltonian $H_{\mathrm{L}}$ is described in the harmonic approximation by:

$$
H_{\mathrm{L}}=\frac{m}{2} \sum\left(\dot{u}_{i}\right)^{2}+\frac{m v_{0}^{2}}{2} \sum\left(u_{i+1}-u_{i}\right)^{2},
$$

where $m$ is the mass of the molecule in the chain, $u_{i}$ is the molecule displacement from the equilibrium position and $v_{0}$ is the sound velocity. The lattice constant is chosen as unity.

The interaction between the electron subsystem and the lattice, in the linear approximation with respect to small deviations $u_{i}$, reads

$$
\begin{aligned}
& H_{\mathrm{e}-\mathrm{L}}=\sum_{i \lambda \delta= \pm 1} t_{1}(\delta)\left(u_{i+\delta}-u_{i}\right) c_{i \lambda \sigma}^{+} c_{i+\delta, \lambda \delta}, \\
& t_{1}(\delta)=-t_{1}(-\delta) .
\end{aligned}
$$

In general the above coupling can influence both the ground state and the excitation spectrum. In the preliminary discussion presented here we neglect the former, 
assuming that the strength of the electron-lattice coupling compared with the correlation and exchange energies is still not large enough to introduce a static distortion. The problem of magnons and solitons in the distorted lattice will be published elsewhere [19].

\section{Magnons}

In this Section we define the magnon operators for the two ground states, ferromagnetic without orbital order $|F\rangle$,

$$
|F\rangle: \quad n_{i 1 \sigma}=n_{i 2 \sigma}=\frac{1}{2} n_{\sigma}
$$

and ferromagnetic with orbital order of "antiferromagnetic" type $|F 0\rangle$. For the latter case it is more convenient to introduce the double labeling of the sites distinguishing between the sublattices $(l, 1)$ if $i=2 l$ and $(l, 2)$ if $i=2 l+1$. The electron site occupations for this state are:

$$
\begin{aligned}
|F 0\rangle: \quad n_{l 11 \sigma} & =n_{l 22 \sigma}=n_{11 \sigma} \\
n_{l 12 \sigma} & =n_{l 21 \sigma}=n_{12 \sigma} .
\end{aligned}
$$

\subsection{Magnons for the ground state $|F\rangle$}

A general form of the magnon creation operator is

$$
\beta_{q \nu}^{+}=\sum_{p \lambda \lambda^{\prime}} b_{\lambda \lambda^{\prime}}^{\nu}(p+q, p) c_{p+q, \lambda+}^{+} c_{p \lambda^{\prime}-},
$$

where $\nu$ labels the magnon branches:

$$
c_{p \lambda \sigma}=N^{-\frac{1}{2}} \sum_{j} c_{j \lambda \sigma} \mathrm{e}^{i p j},
$$

$N$ is the number of lattice sites. For low temperatures only acoustic branch is relevant. In RPA one gets the acoustic magnon amplitude in a form:

$$
b_{\lambda \lambda^{\prime}}(p+q, p)=b(p+q, p) \delta_{\lambda \lambda^{\prime}},
$$

where

$$
\begin{aligned}
& b(p+q, p)=\frac{d_{q}}{\varepsilon_{p+q}-\varepsilon_{p}+\Delta-E_{q}}, \\
& \varepsilon_{p}=2 t \cos (p) \\
& \Delta=\frac{1}{2}(U+J)\left(n_{-}-n_{+}\right) .
\end{aligned}
$$


$E_{q}$ is the magnon energy calculated from RPA equation of motion:

$$
\frac{U+J}{2 N} \sum_{p} \frac{n_{p-}-n_{p+q,+}}{\varepsilon_{p+q}-\varepsilon_{p}+\Delta-E_{q}}=1
$$

and the normalization factor $d_{q}$ is

$$
d_{q}=\left[\sum_{p} \frac{n_{p-}-n_{p+q,+}}{\left(\varepsilon_{p+q}-\varepsilon_{p}+\Delta-E_{q}\right)^{2}}\right]^{-\frac{1}{2}} .
$$

In the following we will use the Fourier transforms of the magnon operators:

$$
\beta_{j}^{+}=N^{-\frac{1}{2}} \sum_{q} \mathrm{e}^{-i q j} \beta_{q}^{+}=\sum_{l_{1} \lambda} b\left(l-j, l_{1}-j\right) c_{l_{\lambda}+}^{+} c_{l_{1} \lambda-},
$$

where

$$
b\left(j, j_{1}\right)=\sum_{p q} \mathrm{e}^{i(p+q) j} \mathrm{e}^{-i p j_{1}} b(p+q, p) .
$$

\subsection{Magnons for the $|F 0\rangle$ ground state}

Before defining the magnon operator let us first write electron band energies for $-F 0\rangle$ given in the Hartree-Fock approximation:

$$
E_{k t \lambda \sigma}=\frac{1}{2}\left(E_{1 \lambda \sigma}+E_{2 \lambda \sigma}\right)+(-1)^{t} \sqrt{\Delta(k, s)},
$$

where $\ell=1,2$ labels the bands:

$$
\begin{aligned}
& \Delta(k, \sigma)=\left(E_{1 \lambda \sigma}-E_{2 \lambda \sigma}\right)^{2}+4 \varepsilon_{k}^{2}, \\
& E_{w \lambda \sigma}=U n_{w \lambda,-\sigma}+V\left(n_{w \lambda+}+n_{w \lambda-}\right)-J n_{w \bar{\lambda} \sigma},
\end{aligned}
$$

$\bar{\lambda}=2$ if $\lambda=1$ and $\bar{\lambda}=1$ if $\lambda=2$.

Upstrokes used anywhere in the text have always the meaning explained above.

The Brillouin zone is half as large as the original. The orbital degeneracy (energy does not depend on $\lambda$ ) is a consequence of the neglection of interorbital hopping (2). The transformation diagonalizing the Hartree-Fock approximation of Hamiltonian (2) reads

$$
a_{k i \lambda \sigma}=\left(\frac{2}{N}\right)^{\frac{1}{2}} \sum_{j w} \mathrm{e}^{i k j} S_{i w}^{\lambda}(k, \sigma) c_{j w \lambda \sigma},
$$

$w=1,2$ labels sublattices and

$$
S_{w w_{1}}^{1}=\left(\begin{array}{cc}
u & v \\
-v & u
\end{array}\right)
$$


with

$$
\begin{aligned}
& u=\left(1+G^{2}\right)^{-\frac{1}{2}} \\
& v=-G\left(1+G^{2}\right)^{-\frac{1}{2}} \\
& G=\frac{1}{2 \varepsilon_{k}}\left(E_{11 \sigma}-E_{21 \sigma}+\sqrt{\Delta(k, \sigma)}\right) .
\end{aligned}
$$

Matrix $S_{w w_{1}}^{2}$ has $u$ and $v$ reversed. The acoustic magnon operator for the considered case takes the form:

$$
\beta_{q}^{+}=\sum_{p \lambda w w_{1}} b_{w w_{1}}^{\lambda}(p+q, p) c_{p+q, w \lambda+}^{+} c_{p w_{1} \lambda-} .
$$

The RPA equation of motion for $\beta_{q}^{+}$is equivalent to the following set of equations for the magnon amplitudes:

$$
\begin{aligned}
& \left(\Omega_{w w_{1}}^{\lambda}-E_{q}\right) b_{w w_{1}}^{\lambda}(p+q, p)+\sum_{r \neq w} \varepsilon_{p+q} b_{r w_{1}}^{\lambda}(p+q, p)-\sum_{r \neq w_{1}} \varepsilon_{p} b_{w r}^{\lambda}(p+q, p) \\
& +\delta_{w w_{1}} \frac{2}{N} \sum_{k} A_{w}^{\lambda}(k+q, k) b_{w w_{1}}^{\lambda}(k+q, k)=0
\end{aligned}
$$

where

$$
\begin{aligned}
& \Omega_{w w_{1}}^{\lambda}=U\left(n_{w \lambda-}-n_{w_{1} \lambda_{+}}\right)+V \sum_{\sigma}\left(n_{w \lambda \sigma}-n_{w_{1} \lambda}\right) \\
& +J\left(n_{w_{1} \bar{\lambda}_{-}}-n_{w \bar{\lambda}_{+}}\right), \\
& A_{w}^{\lambda}(k+q, k)=U\left(n_{k+q, w \lambda_{+}}-n_{k w \lambda_{-}}\right)+J\left(n_{k+q, w \bar{\lambda}+}-n_{k w \bar{\lambda}_{-}}\right),
\end{aligned}
$$

where

$$
\begin{aligned}
& n_{k w \lambda \sigma}=\left\langle c_{k w \lambda \sigma}^{+} c_{k w \lambda \sigma}\right\rangle, \\
& n_{w \lambda \sigma}=\frac{2}{N} \sum_{k} n_{k w \lambda \sigma} .
\end{aligned}
$$

Using (17) one can express (22) explictly

$$
\begin{aligned}
& n_{k 11 \sigma}=n_{k 22 \sigma}=u^{2} \Theta\left(E_{k 11 \sigma}-E_{\mathrm{F}}\right)+v^{2} \Theta\left(E_{k 21 \sigma}-E_{\mathrm{F}}\right) \\
& n_{k 12 \sigma}=n_{k 21 \sigma}=v^{2} \Theta\left(E_{k 11 \sigma}-E_{\mathrm{F}}\right)+u^{2} \Theta\left(E_{k 21 \sigma}-E_{\mathrm{F}}\right),
\end{aligned}
$$

where $E_{\mathrm{F}}$ is a Fermi level and $\Theta$ is a step function. It is easy to check that the magnon amplitudes satisfy the following, intuitively clear symmetry relations:

$$
b_{w w_{1}}^{\lambda}(p+q, p)=b \frac{\bar{\lambda}}{w w_{1}}(p+q, p) .
$$


From (19) using (24) one gets the equation (25) determining the acoustic magnon energy spectrum:

$$
\operatorname{det}\left[\Gamma_{w w_{1}}\left(q, E_{q}\right)-\delta_{w w_{1}}\right]=0
$$

where

$$
\Gamma_{w w_{1}}\left(q, E_{q}\right)=\frac{-2}{N} \sum_{p} L_{w w_{1}}\left(p+q, p, E_{q}\right) A_{w}^{1}(p+q, p)
$$

with

$$
L_{w w_{1}}(p+q, p, E)=\frac{(-1)^{w+w_{1}} M_{w w_{1}}\left(p+q, p, E_{q}\right)}{\operatorname{det}\left[M_{i j}\left(p+q, p, E_{q}\right)\right]}
$$

where

$$
\begin{aligned}
& M_{11}=\Omega_{11}^{1}-E_{q}+\frac{\varepsilon_{p+q}^{2}}{\Omega_{21}^{1}-E_{q}}+\frac{\varepsilon_{p}^{2}}{\Omega_{12}^{1}-E_{q}} \\
& M_{22}=\Omega_{22}^{1}-E_{q}+\frac{\varepsilon_{p+q}^{2}}{\Omega_{12}^{1}-E_{q}}+\frac{\varepsilon_{p}^{2}}{\Omega_{21}^{1}-E_{q}} \\
& M_{12}=M_{21}=\varepsilon_{p+q} \varepsilon_{p}\left(\frac{1}{\Omega_{21}^{1}-E_{q}}+\frac{1}{\Omega_{12}^{1}-E_{q}}\right) .
\end{aligned}
$$

The magnon amplitudes corresponding to the solution of equation (25) are

$$
b_{w w}^{1}(p+q, p)=D_{q} B_{w}(p+q, p)
$$

where $D_{q}$ is a normalization factor and

$$
B_{w}(p+q, p)=\frac{2}{N}\left[L_{w 1}\left(p+q, p, E_{q}\right)+L_{w 2}\left(p+q, p, E_{q}\right) \frac{\Gamma_{11}\left(q, E_{q}\right)-1}{\Gamma_{12}\left(q, E_{q}\right)}\right]
$$

and for $w \neq w_{1}$

$$
b_{w w_{1}}^{1}(p+q, p)=\frac{\varepsilon_{p+q} b_{w_{1} w_{1}}^{1}(p+q, p)-\varepsilon_{p} b_{w w}^{1}(p+q, p)}{\Omega_{w w_{1}}^{1}-E_{q}} .
$$

The amplitudes for $\lambda=2$ are given by (24). Later we shall need the Fourier transforms of magnon operators:

$$
\beta_{j}^{+}=\left(\frac{2}{N}\right)^{\frac{1}{2}} \sum_{q} \mathrm{e}^{-i g j} \beta_{q}^{+}=\sum_{l r l_{1} r_{1} \lambda} b_{r r_{1}}^{\lambda}\left(l r-j, l r_{1}-j\right) c_{l r \lambda+}^{+} c_{l_{1} r_{1} \lambda-},
$$

where

$$
b_{r r_{1}}^{\lambda}\left(l, l_{1}\right)=\sum_{p q} \mathrm{e}^{i(p+q) l} \mathrm{e}^{-i p l_{1}} b_{r r_{1}}^{\lambda}(p+q, p) .
$$

To make the definition (32) unambigous let us allow $j$ to run over the sites of sublattice 1 , i.e. $j=(j, 1)$. 


\section{Magnon-lattice coupling}

A very useful tool to study magnon-magnon or magnon-phonon interactions is the effective magnon Hamiltonian [20] being in analogy to the well-known Holstein-Primakoff boson representation of the Heisenberg Hamiltonian [21], an expansion of the Hubbard Hamiltonian in terms of magnon operators $\beta_{q}^{+}, \beta_{q_{1}}$. Up to terms of fourth order $H_{\text {eff }}$ reads

$$
H_{\mathrm{eff}}=\sum_{q q_{1}} A\left(q, q_{1}\right) \beta_{q}^{+} \beta_{q_{1}}+\sum_{k k_{1} q} W\left(k, k_{1}, q\right) \beta_{k+q_{1}}^{+} \beta_{k-q}^{+} \beta_{k_{1}} \beta_{k},
$$

where

$$
\begin{aligned}
& A\left(q, q_{1}\right)=\left\langle 0\left[\beta_{q}\left[H_{\mathrm{e}}+H_{\mathrm{e}-\mathrm{L}}, \beta_{q_{1}}^{+}\right]\right] 0\right\rangle \\
& W\left(k, k_{1}, q\right)=\frac{1}{4}\left\langle 0\left[\beta_{k+q}\left[\beta_{k_{1}-q}\left[\left[H_{\mathrm{e}}, \beta_{k_{1}}^{+}\right], \beta_{k}^{+}\right]\right]\right] 0\right\rangle .
\end{aligned}
$$

As it is easy to check, an analogous expression to (35) but with $H_{\mathrm{e}}$ replaced by $H_{\mathrm{e}-\mathrm{L}}$ vanishes.

Contrary to the 3D systems the magnon interaction term for the linear chain can not be neglected even at low temperatures. The reason is that the magnon bound states, as was shown by Bethe [22], exist for all wavevectors and thus some of them have arbitrary small energy. The itinerant picture discussion of nonlinear effects resulting from magnon-magnon interactions is under consideration and will be published elsewhere [19]. Here we concentrate only on the nonlineareffects having as a source a coupling of magnetization and lattice fluctuations. The magnon-lattice coupling does not mix the states with different magnon occupations and therefore in a first approximation one can discuss the coupling of magnon with the lattice indepedently from the interaction of magnon bound states with the lattice. In the following we consider only the former problem. We use the free magnon representation since at low temperatures magnons are not strongly influenced by the occurence of magnon bound states due to the small occupation of all possible excitations. Chosing as the ground state $|0\rangle=|F\rangle$ and restricting to the one-magnon subspace one can rewrite $H_{\text {eff }}$ in the more convenient for the present purposes site representation as follows:

$$
H_{\mathrm{eff}}=\sum_{j \Delta} A(\Delta) \beta_{j}^{+} \beta_{j+\Delta}+\sum_{i j \Delta \delta} X_{i-j}^{\Delta \delta}\left(u_{i+\delta}-u_{i}\right) \beta_{j}^{+} \beta_{j+\Delta},
$$

where

$$
\begin{aligned}
& A(\Delta)=\sum_{q} 2 E_{q} \cos (q \Delta) \\
& X_{r}^{\Delta \delta}=t_{1}(\delta)\left(n_{-}-n_{+}\right) \sum_{s}\left[b(r, s) b^{*}(r+\delta-\Delta, s-\Delta)\right. \\
& \left.-b(s, r+\delta) b^{*}(s-\Delta, r-\Delta)\right]
\end{aligned}
$$


and the indices run over: $\delta= \pm 1 ; \Delta=0, \pm 1, \ldots ; r=i-j=0,1, \ldots$

From (4b) and the obvious symmetry relation:

$$
b\left(j, j_{1}\right)=b\left(-j,-j_{1}\right)
$$

results

$$
X_{r}^{\Delta \delta}=-X_{-r}^{-\Delta, \delta} .
$$

A similar effective Hamiltonian for $|0\rangle=|F 0\rangle$ has the form:

$$
\begin{aligned}
& H_{\mathrm{eff}}=\sum_{j \Delta} A(\Delta) \beta_{j}^{+} \beta_{j+\Delta}+\sum_{i t j \Delta \delta} X_{i t-j}^{\Delta \delta}\left(u_{i t+\delta}-u_{i t}\right) \beta_{j}^{+} \beta_{j+\Delta} \\
& X_{i t-j}^{\Delta \delta}=t_{1}(\delta) \sum_{s w \lambda}\left[b_{t w}^{\lambda}(i t-j, s w-j) b_{t w}^{* \lambda}(i t-j+\delta-\Delta, s w-j-\Delta)\right. \\
& \times\left(n_{w \lambda-}-n_{t \lambda+}\right)-b_{w t}^{\lambda}(s w-j, i t-j+\delta) b_{w t}^{* \lambda} \\
& \left.\times(s w-j-\Delta, i t-j-\Delta)\left(n_{t \lambda}-n_{w \lambda+}\right)\right]
\end{aligned}
$$

where: $\Delta=0, \pm 2, \ldots ; \delta= \pm 1 ; i 1-j=0, \pm 2, \ldots ; i 2-j= \pm 1, \pm 3, \ldots$;

\section{Solitary magnons}

The following derivation of the nonlinear Schrödinger equation describing the long wavelength dynamics of the considered system is only a simple generalization of the derivation given by Davydov [1] for molecular systems and adapted by Pushkarov [4] for Heisenberg model. The differences which the reader will see in some intermediate steps are a consequence of the different space extension of the "magnon hopping term" $\left(A(\Delta) \beta_{j}^{+} \beta_{j+\Delta}\right)$ and magnon lattice coupling. In the present considerations they extend beyond the nearest neighbors.

\subsection{Solitons for the ferromagnetic ground state $|F\rangle$}

Using (40) one can rewrite the effective Hamiltonian (36) extended by a lattice part as follows:

$$
\begin{aligned}
& H=H_{\mathrm{L}}+\sum_{j \Delta} A(\Delta) \beta_{j}^{+} \beta_{j+\Delta}+\frac{1}{2} \sum_{j \Delta r \delta} X_{r}^{\Delta \delta}\left[\left(u_{j+r+\delta}-u_{j+r}\right) \beta_{j}^{+} \beta_{j+\Delta}\right. \\
& \left.-\left(u_{j-r-\delta}-u_{j-r}\right) \beta_{j}^{+} \beta_{j-\Delta}\right] .
\end{aligned}
$$

We look for the solution of the Schrödinger equation

$$
\mathrm{i} \hbar \frac{\partial}{\partial t}|\Psi\rangle=H|\Psi\rangle
$$

in the form:

$$
|\Psi(t)\rangle=\sum_{j} C_{j}(t) \beta_{j}^{+}|F\rangle
$$


with the normalization condition:

$$
\langle\Psi(t) \mid \Psi(t)\rangle=\sum_{j}\left|C_{j}(t)\right|^{2}=1 .
$$

The set of equations for the amplitudes $C_{j}$ corresponding to (44) takes the form:

$$
\begin{aligned}
& \mathrm{i} \hbar \frac{\partial C_{j}}{\partial t}=\left[H_{\mathrm{L}}+A(0)+2 \sum_{\Delta>0} A(\Delta)\right] C_{j}+\sum_{\Delta>0} A(\Delta)\left(C_{j+\Delta}+C_{j-\Delta}-2 C_{j}\right) \\
& +\frac{1}{2} \sum_{r \Delta d} X_{r}^{\Delta \delta}\left[\left(u_{j+r+\delta}-u_{j+r}\right) C_{j+\Delta}-\left(u_{j-r-\delta}-u_{j-r}\right) C_{j-\Delta}\right] .
\end{aligned}
$$

To discuss the lattice dynamics the elastic terms should be extended by the magnon-lattice coupling contribution. Following [1] we construct the functional $F(t)=\langle\Psi(t)|H| \Psi(t)\rangle$ playing the role of the Hamilton function in terms of $u_{j}$ and $m \dot{u}_{j}$ with $C_{j}$ fixed. From the Hamilton equations:

$$
\begin{aligned}
& \dot{p}_{j}=-\frac{\partial F}{\partial u_{j}}, \\
& \dot{u}_{j}=\frac{p_{j}}{m}
\end{aligned}
$$

follows

$$
\begin{aligned}
& m \ddot{u}_{j}=m v_{0}^{2}\left(u_{j+1}+u_{j-1}-2 u_{j}\right)+\frac{1}{2} \sum_{r \Delta \delta} X_{r}^{\Delta \delta}\left(C_{j+r+\delta}^{*} C_{j+r+\delta-\Delta}\right. \\
& \left.-C_{j+r}^{*} C_{j+r-\Delta}-C_{j-r-\delta}^{*} C_{j-r-\delta+\Delta}+C_{j-r}^{*} C_{j-r+\Delta}\right) .
\end{aligned}
$$

We are interested in the case when the deformation region is much larger than the lattice constant. We can consider $u_{j}$ and $C_{j}$ as smooth functions of the position, and going over to a continuum approximation:

$$
u_{j}(t) \Rightarrow u(\zeta, t), \quad C_{j}(t) \Rightarrow C(\zeta, t),
$$

(50) and (47) take the form:

$$
\frac{\partial^{2} u(\zeta, t)}{\partial t^{2}}=v_{0}^{2} \frac{\partial^{2} u(\zeta, t)}{\partial \zeta^{2}}+\frac{\Gamma}{m} \frac{\partial}{\partial \zeta}|C(\zeta, t)|^{2},
$$

where

$$
\begin{aligned}
& \Gamma=\sum_{r \Delta \delta} X_{r}^{\Delta \delta} \delta, \\
& \mathrm{i} \hbar \frac{\partial C(\zeta, t)}{\partial t}=B_{1} C(\zeta, t)-B_{2} \frac{\partial^{2} C(\zeta, t)}{\partial \zeta^{2}}+\frac{\Gamma}{m} \frac{\partial u(\zeta, t)}{\partial \zeta} C(\zeta, t),
\end{aligned}
$$

where

$$
B_{1}=H_{\mathrm{L}}
$$




$$
B_{2}=-\sum_{\Delta>0} A(\Delta) \Delta^{2} .
$$

We attempt to find the stationary profile solutions:

$$
\begin{aligned}
& u=u(\zeta-v t), \\
& C=C_{0}(\zeta-v t) \exp [\mathrm{i} \vartheta(\zeta, t)] .
\end{aligned}
$$

It is easy to check that the lattice energy $B_{1}$ does not depend on time

$$
\begin{aligned}
& B_{1}=\frac{1}{2} m v_{0}^{2}\left(1+\frac{v^{2}}{v_{0}^{2}}\right) \int\left(\frac{\partial u(\xi)}{\partial \xi}\right)^{2} \mathrm{~d} \xi, \\
& \xi=\xi-v t .
\end{aligned}
$$

The substitution of (55) into (51) gives

$$
\frac{\partial u}{\partial \zeta}=\frac{-\Gamma}{m\left(v_{0}^{2}-v^{2}\right)}|C(\zeta, t)|^{2} .
$$

Putting now (57) into (53) one gets the well-known nonlinear Schrödinger equation for the magnetization amplitude $C$ :

$$
\mathrm{i} \hbar \frac{\partial C(\zeta, t)}{\partial t}-B_{1} C(\zeta, t)+B_{2} \frac{\partial^{2} C(\zeta, t)}{\partial \zeta^{2}}+B_{3}|C(\zeta, t)|^{2} C(\zeta, t)=0
$$

where

$$
B_{3}=\frac{\Gamma^{2}}{m\left(v_{0}^{2}-v^{2}\right)}, \quad v<v_{0} .
$$

The one-soliton solution to (58) normalized to unity is given by:

$$
C(\zeta, t)=(2 L)^{-\frac{1}{2}} \frac{\exp \left\{\mathrm{i}\left[\frac{h v}{2 B_{2}}\left(\zeta-\zeta_{0}\right)-\Omega t\right]\right\}}{\cosh \left[\frac{\zeta-\zeta_{0}-v t}{L}\right]}
$$

where

$$
\begin{aligned}
& L(v)=\frac{4 B_{2}}{B_{3}(v)}, \\
& h \Omega=B_{1}+\frac{h v^{2}-B_{3}^{2} / 4}{4 B_{2}} .
\end{aligned}
$$

The variable $\zeta_{0}$ appears as a result of the translational invariance of the problem and can be determined by the initial conditions. It should be noted that (60) describes properly only slow moving solitons $\left(v<v_{0}\right)$. In the $v \rightarrow v_{0}$ limit anharmonic effects in the lattice oscillations must be allowed for (for detail see [8]). The low velocity soliton is the more localized the larger the electron-phonon coupling is and the smaller are the elasticity and spin wave stiffness constants. It is easy 
to see that for a vanishing electron-lattice coupling $(\Gamma \rightarrow 0)(60)$ takes the plane wave form.

The soliton-like magnetization deviation (60) is accompanied by the lattice distortion:

$$
\frac{\partial u}{\partial \zeta}=\frac{-\Gamma}{2 m L\left(v_{0}^{2}-v^{2}\right)} \frac{1}{\cosh ^{2}\left(\frac{\zeta-\zeta_{0}-v t}{L}\right)} .
$$

\subsection{Solitons for $|F 0\rangle$ ground state}

The general form of magnon-lattice coupling for the two sublattice case (43) is far more complicated than the one discussed above. Fortunately we are interested here as in the whole paper only in the low energy excitations extending over many lattice sites. In this case it is justified to restrict a discussion to the coupling of magnons with the long wavelength acoustic lattice modes only. Having this in mind one can introduce a reasonable simplifying approximation:

$$
u_{i 1+\delta}-u_{i 1}=u_{i 2+\delta}-u_{i 2},
$$

which transforms the coupling term (43) into the form discussed in the previous Section:

$$
H_{\mathrm{m}-\mathrm{L}}=\sum_{i t j \Delta \delta} X_{i-j}^{\Delta \delta}\left(u_{i t+\delta}-u_{i t}\right) \beta_{j}^{+} \beta_{j+\Delta},
$$

where

$$
X_{i-j}^{\Delta \delta}=\frac{1}{2}\left(X_{i 1-j}^{\Delta \delta}+X_{i 2-j}^{\Delta \delta}\right) .
$$

In this way the present problem is mapped into the one previously discussed. The one-soliton solution has a form (60) with the coupling constants defined by (65) and $\Delta$ running over $0, \pm 2, \ldots$

Summarizing, the present paper gives a formal background for a discussion of solitary bound states of magnons and lattice deformations in the itinerant ferromagnets. In a subsequent paper [19] we will discuss the soliton like magnon bound states and their interaction with the lattice.

\section{Acknowledgments}

The author would like to express his sincere thanks to Professor J. Morkowski for useful discussions.

\section{References}

[1] A.S. Davydov, N.I. Kislukha, Phys. Status Solidi B 59, 465 (1973). 
[2] J.A. Krumhansl, J.R. Schrieffer, Phys. Rev. B 11, 3535 (1975).

[3] M. Lakshman, Th.W. Ruijgrok, C.J.Thompson, Physica A 84, 577 (1977).

[4] D.I. Pushkarov, Kh.I. Pushkarov, Phys. Status Solidi B 81, 703 (1977); Phys. Letts. A 61, 339 (1977).

[5] J. Tjon, J. Wright, Phys. Rev. B 15, 3470 (1977).

[6] J. Corones, Phys. Rev. B 16, 1763 (1977).

[7] U. Lindner, V.K. Fedyanin, Phys. Status Solidi B 89, 123 (1978).

[8] A.S. Davydov, A.V. Zolotaniuk, Phys. Status Solidi B 115, 115 (1985).

[9] D.W Brown, B.J. West, K. Lindenberg, Phys. Rev A 33, 4110 (1986).

[10] J.B. Torrance et al., J. Appl. Phys. 63, 2962 (1988).

[11] P.B. Bagus, J.B. Torrance, Phys. Rev. B 39, 7301 (1989).

[12] A.S. Davydov, Solitons in Molecular Systems, Naukova Dumka, Kiev 1984.

[13] E. Lieb, F. Wu, Phys. Rev. Letts. 20, 1445 (1968).

[14] W. Gill, D.J. Scalapino, Phys. Rev. B 35, 115 (1987).

[15] L.M. Roth, Phys. Rev. 149, 306 (1966).

[16] K.I. Kugel, D.I. Khomski, Zh. Eksp. Teor. Fiz. 64, 1429 (1973).

[17] M. Cyrot, C. Lyon-Caen, Solid State Commun. 21, 837 (1977); J. Phys. (France) 36, 254 (1975).

[18] W.H. Steeb, Phys. Letts. A 55, 433 (1976).

[19] S. Lipiński, to be published.

[20] J. Morkowski, Acta Phys. Pol. A43, 809 (1973).

[21] T. Holstein, H. Primakoff, Phys. Rev. 58, 1098 (1940).

[22] H.A. Bethe, Z. Phys. 61, 205 (1931). 